\title{
11th GCC Closed Forum: cumulative stability; matrix stability; immunogenicity assays; laboratory manuals; biosimilars; chiral methods; hybrid LBA/LCMS assays; fit-for-purpose validation; China Food and Drug Administration bioanalytical method validation
}

Rafiq Islam', Chad Briscoe², Joseph Bower ${ }^{3}$, Stephanie Cape ${ }^{4}$, Mark Arnold $^{5}$, Roger Hayes ${ }^{6}$, Mark Warren7, Shane Karnik ${ }^{8}$, Bruce Stouffer ${ }^{9}$, Yi Qun Xiao ${ }^{10}$, Barry van der Strate ${ }^{11}$, Daniel Sikkema ${ }^{12}$, Xinping Fang ${ }^{13}$, Ariana Tudoroniu ${ }^{14}$, Rabab Tayyem ${ }^{15}$, Ashley Brant ${ }^{16}$, Franklin Spriggs ${ }^{16}$, Colin Barry ${ }^{17}$, Masood Khan ${ }^{17}$, Anahita Keyhani ${ }^{18}$, Jennifer Zimmer ${ }^{19}$, Maria Cruz Caturla ${ }^{20}$, Philippe Couerbe Ardeshir Khadang ${ }^{21}$, James Bourdage ${ }^{22}$, Jim Datin ${ }^{23}$, Jennifer Zemo ${ }^{23}$, Nicola Hughes ${ }^{24}$, Saadya Fatmi ${ }^{25}$ Curtis Sheldon', Scott Fountain ${ }^{26}$, Christina Satterwhite ${ }^{26}$, Kelly Colletti26, Jenifer Vija ${ }^{27}$, Mathilde Yu ${ }^{28}$, John Stamatopoulos ${ }^{29}$, Jenny Lin ${ }^{30}$, Jim Wilfahrt ${ }^{31}$, Andrew Dinan ${ }^{32}$, Susan Ohorodnik ${ }^{10}$, James Hulse ${ }^{33}$, Vimal Patel ${ }^{34}$, Wei Garofolo*,35, Natasha Savoie ${ }^{35}$, Michael Brown ${ }^{36}$, Damon Papac ${ }^{37}$, Mike Buonarati ${ }^{38}$, George Hristopoulos ${ }^{38}$, Chris Beaver ${ }^{39}$, Nadine Boudreau ${ }^{39}$, Clark Williard ${ }^{40}$, Yansheng Liu ${ }^{41}$, Gene Ray ${ }^{41}$, Dominic Warrino ${ }^{41}$, Allan Xu², Rachel Green ${ }^{43}$, Joanne Hayward-Sewell ${ }^{43}$, John Marcelletti ${ }^{44}$, Christina Sanchez ${ }^{44}$, Michael Kennedy ${ }^{44}$, Jessica St Charles ${ }^{6}$, Mohammed Bouhajib $^{45}$, Corey Nehls ${ }^{46}$, Edward Tabler $^{9}$, Jing Tu ${ }^{9}$, Philip Joyce ${ }^{47}$, Adriana Iordachescu ${ }^{48}$, Ira DuBey ${ }^{49}$, John Lindsay ${ }^{50}$, Jim Yamashita ${ }^{50}$ \& Edward Wells ${ }^{51}$

Author affiliations on last page

Disclosure: Due to the equality principles of Global CRO Council for Bioanalysis (GCC), the authors are presented in alphabetical order of member company name, with the exception of the first thirteen authors who provided major contributions to the topics discussed as the topic leaders of the meeting.

The 11th Global CRO Council Closed Forum was held in Universal City, CA, USA on 3 April 2017. Representatives from international CRO members offering bioanalytical services were in attendance in order to discuss scientific and regulatory issues specific to bioanalysis. The second CRO-Pharma Scientific Interchange Meeting was held on 7 April 2017, which included Pharma representatives' sharing perspectives on the topics discussed earlier in the week with the CRO members. The issues discussed at the meetings included cumulative stability evaluations, matrix stability evaluations, the 2016 US FDA Immunogenicity Guidance and recent and unexpected FDA Form 483s on immunogenicity assays, the bioanalytical laboratory's role in writing PK sample collection instructions, biosimilars, CRO perspectives on the use of chiral versus achiral methods, hybrid LBA/LCMS assays, applications of fit-for-purpose validation and, at the Global CRO Council Closed Forum only, the status and trend of current regulated bioanalytical practice in China under CFDA's new BMV policy. Conclusions from discussions of these topics at both meetings are included in this report.

First draft submitted: 4 December 2017; Accepted for publication: 16 January 2018; Published online: 27 April 2018

The Global CRO Council in Bioanalysis (GCC) held its 11th Closed Forum on 3 April 2017 in Universal City, CA, USA. The GCC was founded in 2010 to unite CRO bioanalytical representatives and allow them to share their experiences and opinions related to scientific and regulatory issues in bioanalysis [1]. Topics put forth by member companies are discussed in an effort to provide insight and consensus in the form of conference reports [2-8] and white paper recommendations [9-14] to the bioanalytical community.

The below nine topics were discussed at the 11th GCC Closed Forum, each led by the topic leaders listed 
below. Surveys on each topic were designed by the topic leaders, circulated among GCC members and Pharma representatives prior to the meeting, the results compiled, and then presented in order to begin the discussion of each topic.

- Cumulative stability evaluations - Stephanie Cape

- Matrix stability evaluations - Rafiq Islam

- 2016 US FDA Immunogenicity Guidance - Joe Bower and Bruce Stouffer

- Bioanalytical laboratory's role in writing PK sample collection instructions - Roger Hayes, Mark Warren and Shane Karnik

- Biosimilars - Regulatory Feedback - Rafiq Islam, Yi Qun Xiao, Barry van der Strate and Daniel Sikkema

- CRO perspectives on the use of chiral versus achiral methods - Chad Briscoe

- Hybrid ligand-binding assay (LBA)/LCMS assays - Mark Arnold

- Applications of fit-for-purpose (FFP) validation - Chad Briscoe and Joe Bower

- Bioanalytical Method Validation in China under the China Food and Drug Administration's (CFDA's) new bioanalytical method validation (BMV) policy - XinPing Fang

The second CRO-Pharma Scientific Interchange Meeting was held a few days later in the same location on 7 April 2017. All the above topics (except the China BMV topic) were presented to grant an opportunity for Pharma companies to provide input and feedback and an open dialog between GCC members and Pharma representatives.

This report summarizes all survey results and discussions from both meetings.

\section{Discussion topics}

Cumulative stability evaluations

Fully validated bioanalytical methods require that stability conditions anticipated for incurred samples to be assessed and reported as part of regulatory bioanalytical submissions. Requirements for stability assessments have been established as part of the European Medicines Agency (EMA) and FDA BMV Guidance/Guidelines [15,16]. A survey was circulated to GCC members and Pharma companies to assess the current practices, as well as industry perspectives regarding stability testing, focusing on practices within a regulated bioanalytical laboratory. A total of 50 GCC member representatives and 45 Pharma representatives responded to the survey.

The first set of questions was designed to determine how respondents actually perform stability assessments along with tracking the conditions of known samples and stock solutions. Twice the number of CROs compared with Pharma companies track the exposure to bench top conditions of bulk standards and quality controls (QCs) (52\%

\section{Table 1. Cumulative stability of sample or material evaluation against established stability.}

\begin{tabular}{|llllll|}
\hline $\begin{array}{l}\text { Samples or } \\
\text { materials }\end{array}$ & \multicolumn{2}{c}{$\begin{array}{l}\text { CROs } \\
\text { Believe cumulative } \\
\text { stability is } \\
\text { necessary (\%) }\end{array}$} & $\begin{array}{l}\text { Evaluate cumulative } \\
\text { stability (\%) }\end{array}$ & $\begin{array}{l}\text { Believe cumulative } \\
\text { stability is } \\
\text { necessary (\%) }\end{array}$ & $\begin{array}{l}\text { Evaluate cumulative } \\
\text { stability (\%) }\end{array}$ \\
Incurred samples & 85 & 71 & 62 & 55 \\
\hline Standards and QCs & 65 & 53 & 44 & 34 \\
\hline Stock solutions & 55 & 36 & 35 & 28 \\
\hline Reference material & 30 & NA & 63 & 28 & NA \\
\hline Sample extracts & 53 & NA & 37 & 56 \\
\hline Blank matrices & 8 & & 11 & NA \\
\hline QC: Quality control. & & & & \\
\hline
\end{tabular}


vs $25 \%$ ) and stock solutions (32\% vs $16 \%)$. Most pharma respondents do not track these at all (63\% for known samples and $80 \%$ for stocks), or not with extensive detail, compared with CROs (38\% for known samples and 62\% for stocks). Comments suggest that those who do not track exposure use single use aliquots of calibrants, QCs and stocks, or prepare them fresh daily. For both known samples and stock solutions, those Pharma companies that rely on CROs for their regulated bioanalysis expect some level of tracking if samples and solutions are reused.

A similar question was asked regarding the stability of blank matrices, reference materials and processed extracts. Most CRO and Pharma representatives indicated that they do not typically track exposure to bench top conditions for blank matrices $(85 \%)$ or reference materials $(76 \%)$. However, respondents typically do track exposure for processed extracts (78\% of Pharma and 94\% of CROs).

When tracking exposure to bench top conditions, a paper-based tracking system is used by $22 \%$ of CROs and $33 \%$ of Pharma, while an electronic system is used by $64 \%$ of CROs and $28 \%$ of Pharma. A combination of both may also be used (10\% CROs, 6\% Pharma).

Exposure to bench top conditions is considered irrelevant when single-use aliquots are utilized. CRO and Pharma laboratories similarly utilize single-use aliquots of blank matrix at least occasionally, if not routinely, only $40 \%$ of the time. Standards and QCs are divided into single-use aliquots $90 \%$ of the time by both CROs and Pharma. CROs use single-use stock solutions somewhat more frequently $(80 \%)$ than Pharma companies (68\%).

Both CROs and Pharma companies were asked if they believed that the time at conditions outside of routine storage should be considered cumulatively and evaluated against established stability for incurred samples, known samples (standards and QCs), stock solutions, reference materials, sample extracts and blank matrices. Only $14 \%$ of CROs and $20 \%$ of Pharma did not think this was necessary for any of these types of samples and materials. Table 1 outlines the percentage of CRO and Pharma respondents who believe that cumulative stability should be evaluated against established stability for certain types of samples or materials, as well as how many actually do the evaluation. It is interesting to note that $77 \%$ of all respondents have not received any direct regulatory feedback that exposure time must be considered cumulatively.

Results demonstrate that overall, respondents and attendees did believe there was value in tracking cumulative stability of some types of samples. However, a lower number of respondents were actually calculating cumulative exposure. It was suggested that this may be due to the fact that electronic laboratory notebooks (ELN) are not typically designed to perform cumulative tracking. Bioanalytical representatives suggest encouraging vendors to add this feature to their products to facilitate tracking this information. Furthermore, there is less of a need for tracking cumulative stability if validation exercises are performed for long durations (e.g., bench top stability proven for $24-48 \mathrm{~h}$ ) so that detailed tracking is only required for less stable molecules or if proven durations are short.

\section{Matrix stability evaluations}

A Health Canada Notice was released in October 2015 [17] and amended in March 2016 [18] requiring that matrixbased stability experiments be assessed using three individual tubes of QC samples that underwent exposure conditions. Attendees informed the GCC membership that deficiencies were also being received requesting that the whole blood stability be tested in this way. Discussions concerning bioanalytical laboratories' reactions to this Notice began at the 10th GCC Closed Forum [8], occurred as part of the 2016 and 2017 Workshop on Recent Issues in Bioanalysis $[19,20]$ and continued at this Forum. A survey released to CRO and Pharma participants indicated that, prior to this Notice, $70 \%$ of respondents exposed a single tube to stability conditions and then assayed at least three replicates from this tube. This practice was deemed scientifically sound by $90 \%$ of respondents, because the potential of stability being related to tube-to-tube variability of known samples during stability testing is either none, too small to measure, or not significant enough to measure with $\mathrm{N}=3$.

Attendees were asked if, when repeating stability testing in order to conform to Health Canada's requirements, any stability variations between tubes had been detected; $97 \%$ of respondents indicated that they have never seen any evidence of the number of tubes impacting stability results. Since, QC pools are homogeneous, there is no scientific expectation of any significant difference between results using one tube versus three tubes. Many laboratories have provided stability data in an effort to compile data-driven, scientific proof to dissuade this opinion. Any laboratory wanting to provide additional data is encouraged to contact the GCC.

\section{FDA immunogenicity guidance}

The FDA released a draft immunogenicity guidance document in April 2016 [21]. Despite it being a draft document, GCC members indicated that several regulatory findings were issued because of lack of compliance with the regu- 
latory thinking contained in the document. These deficiencies related to cut points (confirmatory cut point [CP] CI should be $99 \%$ and not $99.9 \%$ ), increased requirements (e.g., precision of positive controls [PCs], interference testing, hemolysis) for Tier 2 validations, and the need for NAb assays to have low QCs close to the CP. In order to determine how widespread these inspectional findings are, a survey was sent to the membership seeking feedback.

Respondents indicated that less than $25 \%$ of bioanalytical laboratories have been inspected for an immunogenicity study in the last 24 months. Of those who have been inspected, $20 \%$ of CROs and $67 \%$ of Pharma have received a finding related to anti-drug antibody (ADA) assays, $13 \%$ of CROs and $33 \%$ of Pharma have received one for NAb assays and $17 \%$ of Pharma have received one for noncell-based assays. Specific questions related to reported deficiencies were asked.

Regarding Tier 2 confirmatory assay validations, respondents overwhelmingly disclosed that they evaluated the precision of PCs. Hemolysis and lipemia interference tests were done by $40 \%$ of respondents. Selectivity with and without the drug were done by $80 \%$ of CROs and $61 \%$ of Pharma.

When asked what confidence interval is used for cut-point determination of confirmatory assays, approximately $75 \%$ of respondents reported using $99.0 \%$, as required by the draft guidance. Furthermore, the draft guidance requires that the assay sensitivity of ADA methods be $100 \mathrm{ng} / \mathrm{ml}$. When this sensitivity cannot be reached for a method, $40 \%$ of CROs and 30\% of Pharma redeveloped the method. However, $20 \%$ of CROs and $36 \%$ of Pharma elect to justify the appropriateness of the sensitivity instead.

Overwhelmingly, respondents indicated that they recommended the use of affinity purified PCs. A total of $67 \%$ of CROs and $87 \%$ of pharma require a certificate of analysis from their clients for PC antibodies.

The survey also inquired about the availability of certain processes or policies. Approximately, $75 \%$ of total respondents have a procedure for extending PC expiration. The most used approach for this was based on assay performance (100\% of CROs and $74 \%$ of Pharma). All respondents reported having an SOP that allowed for a tiered approach to ADA validation (screen, confirm, titer). Only half of respondents have an SOP for the validation of NAb assays. The update of SOPs to include recommendations from the draft 2016 guidance varied between CROs and Pharma. A total of $60 \%$ of CROs reported that they have updated their SOPs accordingly, whereas only $33 \%$ of Pharma respondents have done so.

\section{Bioanalytical laboratory's role in writing PK sample collection instructions}

Part of the bioanalytical laboratory's role in method validation is to determine and assess sample stability conditions in order to ensure that sample integrity remains intact. However, many critical steps required to maintain sample integrity occur at the clinical/nonclinical facility (collection, processing and temporary storage) and during the shipment of samples to the laboratory. In order to ensure that the proper steps are taken, laboratory manuals can be written to provide sample collection and shipping instructions to clinical/nonclinical facilities. Therefore, a survey was circulated in order to determine current industry practice with $53 \mathrm{CRO}$ and 52 Pharma respondents who perform either clinical or nonclinical bioanalysis, or both.

It is not always clear who has the responsibility to prepare this manual. Over $60 \%$ of CROs indicated that they were often or always required to provide PK sample collection instructions, compared with only $35 \%$ of Pharma companies, which stated that they request the bioanalytical laboratories to provide them. When the laboratories were asked who was requesting the instructions, respondents indicated that it was the sponsors (77\%), the clinical site (58\%), the nonclinical test facility (37\%) and the central laboratory (22\%). Approximately, 9\% stated that they sent the instructions proactively, without a request. Interestingly, results were very different for the Pharma respondents who stated that they provide instructions $66 \%$ of the time, request from central laboratories $41 \%$ of the time, and clinical sites and nonclinical test facilities $11 \%$ of the time.

Stability data is available about $70 \%$ of the time for the test article prior to providing sample collection instructions, according to all respondents. CROs further indicated that $52 \%$ of the time they rarely or never receive details of actual sample handling conditions from the in-life sites, and only about $25 \%$ claim to receive them often or always. Fortunately, respondents indicated that in-life facilities followed the PK sample instructions at least most of the time (68\% for CROs and $48 \%$ for Pharma). CROs even indicated this was always the case $30 \%$ of the time, versus $50 \%$ for Pharma. Although not unanimous, $75 \%$ of CROs and $70 \%$ of Pharma confirmed that bioanalysis laboratories were not responsible for ensuring that the in-life facility followed the instructions.

When asked if respondents have ever encountered the sample container having an impact on sample analysis, $70 \%$ of CROs and $57 \%$ of Pharma stated that they had rarely or never seen this situation. The remaining respondents had only seen it occasionally. CROs had no consensus on who determines and provides supplies to be used for sample collection. The results were split evenly between the in-life facilities, the bioanalytical laboratories, 
the sponsors and the central laboratories. Interestingly, a small number of Pharma respondents indicated that the bioanalytical laboratory should provide the supplies (only 15\%). A total of 68\% indicated that it should be the central laboratory, $45 \%$ the sponsor and $32 \%$ the in-life facility.

It was the impression that often the bioanalytical laboratory is tasked with reconciling discrepancies regarding sample collection and sample identity, as well as performing additional stability evaluations if excursions are reported. The survey results indicated that, in the case of excursions to sample handling conditions, approximately $90 \%$ of all the respondents confirmed that sponsors should bear the cost of establishing additional stability to support sample integrity. According to CROs, sample collection should be reconciled by the in-life facility $80 \%$ of the time, the central laboratory $53 \%$ of the time, the sponsor $35 \%$ of the time, and the bioanalytical laboratory only $25 \%$ of the time. Similar results were obtained for Pharma companies, except that in-life facilities and central laboratories were responsible according to $52 \%$ of Pharma respondents and clinical laboratories $60 \%$, which is counter to the industry perception that bioanalytical laboratories bear the brunt of reconciliation. Finally, when asked if data transfer agreements should specify that the bioanalytical laboratory will manually edit the study data based on sample collection reconciliation, $45 \%$ of CROs and $35 \%$ of Pharma said yes. Therefore, there is no consensus on the content of these agreements.

Discussions of these results concluded that data transfer agreements should be in place, and at least outline who is responsible for authorizing the changes and modifying the data. Data transfer agreements should also outline what information should be included in the files, so that the bioanalytical laboratory does not find itself handling data for which it is not responsible. Although, the bioanalytical laboratories cannot be responsible for how samples are collected and processed at the in-life facility, they should have procedures for sample reconciliation and resolution of detectable discrepancies prior to the analysis of incurred samples.

\section{Biosimilars}

Several regulatory documents have been released discussing the analysis of biosimilars for both PK and ADA assays [22-25]. Biosimilars were last discussed during the 9th GCC Closed forum in 2015 [7], and it was decided to provide another survey to GCC members to see how industry approaches have evolved in the last 2 years, as well as to evaluate any differences between CRO and Pharma approaches. Responses were obtained from $26 \mathrm{CRO}$ and 18 Pharma representatives. Overall, only $15 \%$ of CRO respondents have had any interaction with regulatory authorities on biosimilar submissions, compared with $45 \%$ of Pharma companies. Several challenges for both PK and ADA assays are faced by bioanalysts equally in both CRO and Pharma laboratories. These include lack of critical reagents, difficulties in method establishment, and the cost and time required for method development.

There was no clear consensus on whether a LBA was sufficient for assaying NAb against biosimilars as opposed to cell-based NAb assays. Only $45 \%$ of CROs and $60 \%$ of Pharma respondents stated that LBAs were sufficient, and most respondents still use cell-based assays. If the biosimilar shows fingerprint-like similarity to its reference drug, the majority of respondents believe the necessity of conducting nonclinical evaluations should be discussed with the regulatory authorities. When asked if nonclinical ADA assays are needed, $45 \%$ of CROs and $25 \%$ of Pharma respondents said no nonclinical ADA assays were needed. Those who responded positively, mostly (45\% both CROs and Pharma respondents) agreed that one assay was sufficient to support ADA analysis for nonclinical studies. For those few using two assays for nonclinical ADA testing, the criteria to use in terms of CP similarity were not harmonized. CROs were equally split between 2-3-fold, 3-4-fold and 80\%-120\% differences and other unspecified criteria. Pharma companies primarily used other unnamed criteria and occasionally $2-3$ fold or $80 \%-120 \%$ differences.

Another area having no consensus was the criteria to establish similarity in clinical ADA results between originators and biosimilars. A majority (55\%) of the Pharma respondents and only $35 \%$ of the CRO respondents utilize $80-125 \%$ criteria. A minority of CROs and Pharma respondents also utilize $70-130 \%$, and $80-120 \%$ criteria to establish similarity. Criteria to determine nonclinical ADA incidence was also different between CRO and Pharma respondents. Pharma claims to primarily use other unnamed criteria, whereas CROs use $70-130 \%$ only a third of the time, followed by $80-120 \%$ and $80-125 \%$.

Regarding clinical biosimilar studies, the most common design used by CROs was the crossover design $(53 \%)$ followed by the parallel design (35\%). The inverse was true for Pharma respondents. The crossover design was used $55 \%$ of the time and the parallel design $27 \%$ of the time. It was concluded during the discussions with attendees that if equivalence was shown during validation, only a parallel design was needed. When determining biosimilarity, total PK assays were used by $47 \%$ of CROs and only $22 \%$ of Pharma. Free assays were used by $36 \%$ of CROs and $11 \%$ of Pharma. Reagent dependent PK assays were used $16 \%$ by CROs and $44 \%$ by Pharma. 
Approaches between both types of bioanalytical laboratories are not harmonized. When queried about the type of matrix used for ADA CP assessments, CROs used healthy subjects most often ( $60 \%$ of the time) whereas Pharma had no preferred method. For biosimilar ADA development and validation, Pharma companies responded that they overwhelmingly used one assay. CROs were split between both one assay and two assays (biosimilar and US originator). When deciding on what to use as the positive control, half of CROs and Pharma used the PC against the biosimilar, approximately $30 \%$ used the PC against the originator and the remaining used both the biosimilar and the originator. Overwhelmingly, respondents used one assay for quantifying biosimilar/originator materials. The criteria most often used to demonstrate that the method is comparable for measuring biosimilar and originator samples is $\pm 20 \%$. There was no consensus as to whether the evaluation of the binding characteristics of assay reagents to both biosimilars and reference products was required. Pharma respondents only evaluated this half the time, whereas $90 \%$ of CROs did not perform this evaluation at all.

\section{CRO perspective on the use of chiral versus achiral methods}

Recently, chiral assays have received increased scrutiny from regulatory agencies. There have been inconsistencies observed between the global regulatory agencies expectations both in the necessity of chiral assays and the expectations for the performance of those assays. Chiral assays are expensive and often challenging because they may require long development time and may not be rugged in application. Through survey and discussion between GCC and GCC Pharma Exchange Partners, some of the key topics related to the application of chiral assays in various stages of drug development, including bioequivalence (BE) studies, were assessed.

When asked if chiral assays are used to support BE studies for enantiomerically pure drugs, results were similar between CROs and Pharma respondents. The majority stated that a chiral assay is not required if it can be proven that interconversion does not occur. It would only be needed if interconversion could or was likely to occur.

CROs tended to be conservative when asked about when chiral assays were needed for studies which support new drug programs for NDA submission, tending toward chiral assays for all studies or allowing the decision to be sponsor driven. One option adopted by many of the respondents from both CROs and Pharma involved analyzing samples from one study in each species.

In regards to the question of any current regulatory guidance requiring chiral assays, $50 \%$ of respondents felt that the requirement was suggested by the EMA BMV guideline [15], 25\% by the draft FDA BMV guidance [26], and $10 \%$ by the 2001 FDA BMV guidance [16]. CROs and Pharma representatives were then asked if their organization had received a deficiency letter for the use of an achiral assay in a BE submission, and if yes, by which regulatory agency. Five of the 41 respondents confirmed receipt of a total of seven deficiency letters, of which five were from EMA, one was from the FDA and one was from an unspecified agency. It was noted that EMA appeared to be more proactive in the review of new literature. Additionally, Committee for Medicinal Products for Human Use (CHMP) has standing review questions for nonclinical Electronic Common Technical Document packages about chiral centers in a molecule and whether the chiral centers are involved in metabolism.

When conducting a chiral assay with a reference standard identified as a racemic mixture, there was no consensus on whether a determination of the ratio of enantiomers is required. A quarter of CROs and half of Pharma respondents require this in all cases. Another quarter of CROs versus only $1 \%$ of Pharma respondents request the determination, but do not mandate it. If standards and QCs are prepared using the same lot of material, the determination is not required. Most of the remaining respondents stated that enantiomerically pure chiral reference material was used.

Several scientific observations and opinions were queried regarding chiral and achiral assays. Bioanalysts were asked if the sum of the $\mathrm{R}$ and $\mathrm{S}$ enantiomers was equivalent to results obtained with an achiral method. Only $10 \%$ said no, citing Lercanidipin as an example. A total of $95 \%$ have not observed ion suppression between coeluting enantiomers. Finally, 74\% of bioanalysts have not observed ionization differences between $\mathrm{R}$ and $\mathrm{S}$ enantiomers. Almost all (91\%) of respondents stated that interconversion was tested in all cases, or at least if it was probable, during the extraction and in vivo. There was no consensus on whether interconversion should be tested in both directions; $60 \%$ test both directions. Regarding resolution of chiral assays, there was also no consensus on whether a certain amount of resolution was required, and if it should be monitored. Only $65 \%$ specified that resolution was required, but responses varied greatly on how much resolution is required and how it should be monitored/reported. Another area where further discussions are needed to reach harmonization is in regards to what enantiomeric ratios are required when performing precision and accuracy. A total of $32 \%$ of respondents stated it should be performed at equal ratios, $50 \%$ stated at biologically relevant ratios and the remainder believed it should be performed at whatever ratio is present in the commercially available reference standard. Attendees 
concluded during the meeting that if there is no or low in vitro interconversion, then using close ratios is acceptable. However, if there is significant interconversion, then more extreme enantiomeric ratios should be used and monitored during the use of the assay. One way this can be done is to add separate QCs containing individual enantiomers to demonstrate there is no interconversion during sample analysis.

\section{Hybrid LBA/LCMS assays}

In hybrid LBA/LCMS assays, immunoaffinity is used to capture or enrich the target molecule in the sample and then LCMS is applied to selectively detect and measure the analyte. As a relatively new methodology, significant discussions about this topic began only in 2012 [27] and interest continues to grow [19-20,28-31]. In order to assess current practices, as well as industry perspectives regarding these types of assays, a survey was sent which focused on current and future plans for implementation of hybrid LBA/LCMS assays.

Respondents were equally split between CROs and Pharma companies. Out of these, half of each group perform hybrid assays occasionally or routinely and another $22 \%$ of CROs and $12 \%$ of Pharma intend to perform them in the future. Overwhelmingly, CROs believe that the use of hybrid assays will only increase over the next 3 years. Pharma respondents were split between increased or similar use. The CROs that perform hybrid assays used them across all study types; viz., discovery, nonregulated PK studies, both clinical and preclinical regulated PK studies and biomarker studies, although they were used for nonregulated PK studies $10 \%$ more often than the other types. The Pharma respondents typically use hybrid assays more often in the discovery or nonregulated PK space, although $40 \%$ do use them for regulated PK studies and biomarkers. The types of molecules assayed using hybrid LBA/LCMS include therapeutic proteins and peptides. CROs also assayed antibody-drug conjugates using hybrid assays about twice as often as Pharma respondents. Oligonucleotides were assayed with this platform less than $10 \%$ of the time. There was discord between CRO and Pharma respondents regarding requiring certificates of analysis for antibody reagents. Sixty percent of CROs require a certificate of analysis compared with only $30 \%$ of Pharma respondents.

Several different assay approaches were queried. In $75 \%$ of the laboratories, magnetic beads were used for the assay. Coated plates, mass spectrometric immunoassay (tips) and clinical kits were also used, but to a lesser extent. Triple quadrupole mass spectrometers were used $80 \%$ of the time. Manual sample handling was used at least twice as often as automated handling options. CROs source their capture antibodies both from the sponsors and from commercial suppliers. Pharma companies mostly use commercially supplied or in-house produced capture antibodies. In-house processes and materials supplied with the label were both used to achieve biotinylation or other labeling. CROs typically do not have established procedures for qualifying labeling reagents (67\%) compared with only $30 \%$ of Pharma. Capture and detection antibodies are overwhelmingly considered critical reagents and they are characterized as such. When working with proteins $>10 \mathrm{kDa}$, respondents indicated that they mostly use digestion and surrogate peptide quantitation. There was no consensus on the type of internal standard to use for hybrid assays; in other words, SIL or analog full length proteins, as well as SIL and analog peptides, with or without overhanging amino acids, were all used.

When conducting a hybrid LBA/LCMS assay, there was no consensus on what SOP to use; an LBA SOP, an LCMS SOP, a hybrid SOP, or some other procedure. Both CROs and Pharma respondents use assay dependent criteria $40 \%$ of the time and LCMS criteria $20 \%$ of the time. However, there was a significant difference with the use of ligand binding criteria. CROs use LBA criteria $35 \%$ of the time, while Pharma companies only apply it $10 \%$ of the time; Pharma respondents use other criteria instead.

Further experience with this methodology is needed in order to harmonize issues that have yet to reach consensus.

\section{Applications of fit-for-purpose validation}

There are a variety of reasons why bioanalytical assays may not meet the definition of a full method validation as outlined in the various BMV guidelines [15,16,32] due to, for example, insufficiently characterized reference standards or inability to demonstrate stability due to matrix type (e.g., tissue). It was concluded during the 7th GCC meeting [5] that a FFP approach should be limited to demonstrating acceptable assay performance when alternatives to challenging assays are not available.

To avoid confusion, at the beginning of the discussion, it was mentioned that on some occasions, FFP validation has also been called 'scientific validation'. However, this new verbiage is not recognized by regulators and by many in the industry who believe that another synonym for FFP validation is not really needed [33]. Hence, only the term 'FFP validation' was used at both the GCC Closed Forum and CRO-Pharma scientific interchange meetings. 
After four more years of experience using and supporting drug submissions with FFP assays, a survey was sent to GCC members and Pharma companies to determine how utilizing a FFP approach has evolved. Approximately $60 \%$ of responding companies label validations that do not meet regulatory definitions of full validation as 'assay qualification'. Interestingly, when asked the respondents' preferred term, the vast majority preferred 'FFP validation'. The types of studies supported by FFP assays are wide-ranging, from non-GLP animal studies to Phase III studies. Pharma respondents tended to use FFP assays more often than CROs for Phase I studies, whereas CROs used FFP assays more than Pharma for Phase III biomarker studies. The respondents overwhelmingly agreed that it is possible to perform a study using a FFP validated assay, obtain meaningful data, and submit the resulting data to a regulatory agency.

When asked if bioanalysts work with early development/pre-clinical groups to develop the most clinically relevant biomarkers or if they prefer to let them request work, responses differed between CROs and Pharma. Pharma groups tended to work closely together to define the relevant biomarkers, which is logical considering both groups typically belong to the same organization. It is no surprise, therefore, that CRO respondents experience a different reality, where only $30 \%$ work closely with the development/pre-clinical groups, $25 \%$ let them request the work that they see fit, and $45 \%$ stated that it depended on the sponsor. FFP assays are qualified within the bioanalytical laboratory at CROs $60 \%$ of the time compared with $30 \%$ of the time in Pharma companies, whereas $55 \%$ of the time, a combination of groups performs these assays.

CROs typically do not have a specific group designated for early research/unregulated biomarker assays, however approximately half of Pharma companies do. When such a group exists, half of them do not follow a formal process or SOP for the transfer of early stage methods. For method transfers between sponsors and CROs, the need for performing bridging experiments was queried. For laboratory-to-laboratory transfers within the same organization, CROs perform bridging experiments approximately $30 \%$ of the time, compared with $10 \%$ of Pharma companies. However, when transferring between CROs, bridging experiments were performed $55 \%$ of the time according to CROs and $70 \%$ of the time according to Pharma respondents.

Only $45 \%$ of CROs and $30 \%$ of Pharma respondents have a specific SOP that covers FFP validation although approximately $70 \%$ of respondents have at least a matrix of required minimum experiments. Despite these differences, approximately $60 \%$ maintain the same level of record keeping for FFP assays as they do for regulated validations, and $80 \%$ submit the documentation through a secondary review process.

Approximately, half of all respondents' validation SOPs cover the validation of rare or tissue matrices. Those respondents whose SOPs do not cover rare or tissue matrices do not have a separate SOP either. When performing validations using rare or tissue matrices, an overwhelming majority apply the FFP validation principles and use the same platforms they would use for normal matrices. The majority of respondents use surrogate matrix calibrants and authentic matrix QCs (74\% of CROs and 60\% of Pharma) or authentic matrix for both calibrants and QCs (23\% of CROs and 22\% of Pharma). Surrogate matrices could be assay buffer, serum/plasma or similar tissue type from a different species. Various approaches are used to determine the acceptance criteria for methods assaying tissue samples. Approximately $45 \%$ of all respondents base the criteria on method performance. BMV criteria are used by $42 \%$ of CROs and $25 \%$ of Pharma, whereas less stringent criteria are used by $14 \%$ of CROs and $28 \%$ of Pharma. Stability assessments for methods in tissue are typically performed in tissue homogenate.

In conclusion, FFP validation and FFP assays are widely used by both CROs and Pharma companies to submit data to regulatory agencies. The decision to apply an FFP approach to various situations is decided on a case by case basis.

\section{Bioanalytical method validation in China under CFDA's new BMV policy}

China's investments into pharmaceutical research and development have not been as extensive as in Western countries, and especially China's late-stage drug development of their Pharma industry has lagged behind the USA and EU. This has led to a lack of experience in late-stage drug development by both Chinese industry and regulators. The majority of bioanalytical work has been done in academic and hospital laboratories with weak regulated processes, reflected in insufficient compliance to quality in drug submissions to the CFDA.

In order to remedy the current regulatory gaps in China, several regulations have been put into place. The Chinese 2015 Pharmacopeia, Section 9012 [34], provides a detailed description of BMV and sample analysis requirements, mainly consistent with the draft US FDA and EMA BMV Guidance/Guidelines [15,26] although it was noted that some of the requirements are even more stringent in China. Also released in 2015 was an announcement requiring Pharma companies to conduct self-inspections of their research data for 1622 submissions [35]. 
This resulted in nearly $83 \%$ of submissions being withdrawn. All generic drugs approved prior to 2007 must also be re-evaluated by a 2018 deadline.

In order to better regulate BE studies going forward, three additional guidelines were released in 2016, one regarding the use of PK parameters in $\mathrm{BE}$ evaluation and two inspection guidelines outlining how $\mathrm{BE}$ studies are inspected at the clinical sites and bioanalytical laboratories $[36,37]$. As a result of these guidelines, CFDA formed a dedicated inspection team and recruited additional inspectors to conduct more frequent on-site inspections. These on-site inspections uncovered many data integrity issues at both clinical sites and bioanalytical laboratories, resulting in old BE study submissions being withdrawn or repeated. Some unqualified bioanalytical laboratories were closed or reorganized.

Only a limited number of clinical sites $(\sim 100)$ were qualified to conduct new standard clinical BE studies in 2015-2016, but the new policy allows $\sim 500$ hospitals to be qualified to perform BE studies. New BE submissions are now being inspected immediately to ensure data reliability, integrity, compliance and traceability from clinical sites and bioanalytical laboratories. Local (provincial team) and central (CFDA's Center for Food and Drug Verification team) dual inspections are being conducted, applying the current regulation requirements to old studies. Once the inspections are complete, the Center for Food and Drug Verification central team meets with the submitting company and CRO representatives to discuss the final inspection conclusions. Then, the Center for Drug Evaluation reviews and makes the final decision for approval or rejection. Attendees expressed high expectations of the improvement of China's related bioanalytical environment and a hope that the Chinese agency would also consider future harmonization with other leading regulatory agencies in order to facilitate international submissions.

\section{Future perspective}

The GCC, as a global organization, will continue to provide recommendations on hot topics of global interest in small and large molecule bioanalysis, biomarkers and immunogenicity. Additionally, CRO-Pharma scientific interchange meetings will continue in order to facilitate communication between the two. Please contact the GCC [38] for the exact date and time of future meetings, and for all membership information.

\section{Acknowledgements}

The GCC would like to thank the following: N Savoie (GCC) for facilitating the 11th GCC Closed Forum; C Briscoe (PRA Health Sciences) for designing the survey, collecting answers, preparing survey results and chairing the sessions on 'GCC perspective on chiral and achiral methods used in bioequivalence studies'; R Islam (Celerion) for designing the survey, collecting answers, preparing survey results and chairing the session on 'GCC perspective on matrix-based stability experiments'; S Cape (Covance) for designing the survey, collecting answers, preparing survey results and chairing the sessions on 'Sample, standard and QC tracking to demonstrate stability within validation parameters' and 'hybrid LBA/LCMS bioanalysis'; M Arnold (Covance) for designing the survey, collecting answers, preparing survey results and chairing the sessions on 'hybrid LBA/LCMS bioanalysis'; J Bower (Covance) and B Stouffer (PPD) for designing the survey, collecting answers, preparing survey results and chairing the sessions on 'GCC Survey on Immunogenicity guidance and 483s'; R Hayes (MPI Research), M Warren (Atlanbio), S Karnik (Pyxant Labs) for designing the survey, collecting answers, preparing survey results and chairing the session on 'Bioanalytical labs' role in writing PK sample collection instructions'; P Bennett (PPD), J Bower (Covance) and C Briscoe (PRA Health Sciences) for designing the survey, collecting answers, preparing survey results on 'Application of fit-for-purpose validations'; J Bower (Covance) and C Briscoe (PRA Health Sciences) for chairing the session on 'Application of fit-for-purpose validations'; R Islam (Celerion), Y Q Xiao (Envigo), B van der Strate (PRA Health Sciences) and D Sikkema (Frontage) for designing the survey, collecting answers, preparing survey results and chairing the session on 'Biosimilars - regulatory feedback'; X Fang (WuXi Apptec) for chairing the session on 'Status and trends of current regulated bioanalytical practice in China under CFDA's new BMV policy'; all the GCC member companies who filled in the numerous surveys, participated and contributed to the discussions at the 11th GCC Closed Forum for Bioanalysis; all the Pharma representatives who filled in the numerous surveys, participated and contributed to the discussions at the 2nd GCC Pharma Scientific Interchange Meeting: J Sydor (Abbvie), C Whiting (Aduro BioTech), M Li (Alexion Pharmaceuticals), J Ma (Alexion Pharmaceuticals), P Cao (Alkermes), D Desai-Krieger (Allergan), C James (Amgen), L Coppola (Apotex), A Wilson (AstraZeneca), E David (Biogen), S Zoog (BioMarin), J Duggan (Boehringer Ingelheim), L Lohr (ChemoCentryx), J Pav (Daiichi Sankyo), L He (Daiichi Sankyo), E Wickremsinhe (Eli Lilly), R Gonthier (EndoCeutics), H Birnboeck (F Hoffmann-La Roche), A Ahene (Five Prime Therapeutics), T Tarnowski (Gilead), B Dean (Genentech), R Rourick (Genentech), C Hottenstein (GlaxoSmithKline), L Abberley (GlaxoSmithKline), M Mikelj (Hexal/Sandoz), J Cao (Ignyta), JM Lecerf (ImmunoGen), S Spitz (Incyte), D Zhou (La Jolla Pharmaceutical Company), S Breidinger (Merck), R Luo (Neurocrine Biosciences), M Fox (NGM Biopharmaceuticals), M Niwa (Nippon Kayaku), S Roth (Otsuka Pharmaceutical), B Gorovits (Pfizer), O Kavetska (Pfizer), 
A Musuku (Pharmascience), C Foerder (Seattle Genetics), J Wu (Shire), YL Chen (Sunovion), M Qian (Takeda); N Savoie (GCC) for taking the minutes of the 11th GCC Closed Forum and drafting the first version of this commentary; W Garofolo (GCC) for organizing the logistics of the meeting and coordinating the review of this commentary.

\section{Financial \& competing interests disclosure}

The authors have no relevant affiliations or financial involvement with any organization or entity with a financial interest in or financial conflict with the subject matter or materials discussed in the manuscript. This includes employment, consultancies, honoraria, stock ownership or options, expert testimony, grants or patents received or pending, or royalties.

No writing assistance was utilized in the production of this manuscript.

\section{References}

1 Premkumar N, Lowes S, Jersey J et al. Formation of a Global Contract Research Organization Council for Bioanalysis. Bioanalysis 2(11), 1797-1800 (2010).

2 Breda N, Garofolo F, Cruz Caturla M et al. The 3rd Global CRO Council for Bioanalysis at the International Reid Bioanalytical Forum. Bioanalysis 3(24), 2721-2727 (2011).

3 Lowes S, Jersey J, Shoup R et al. 4th Global CRO Council for Bioanalysis: coadministered drugs stability, EMA/US FDA guidelines, 483s and carryover. Bioanalysis 4(7), 763-768 (2012).

4 Nicholson R, Lowes S, Cruz Caturla M et al. 6th GCC focus on LBA: critical reagents, positive controls and reference standards; specificity for endogenous compounds; biomarkers; biosimilars. Bioanalysis 4(19), 2335-2342 (2012).

5 Rocci M, Lowes S, Shoup R et al. $7^{\text {th }}$ GCC: use of incurred samples; fit-for-purpose validation; solution stability; electronic lab notebooks; hyperlipidemic matrix testing. Bioanalysis 6(20), 2713-2720 (2014).

6 Bower J, Fast D, Garofolo F et al. $8^{\text {th }}$ GCC: consolidated feedback to US FDA on the 2013 draft FDA guidance on bioanalytical method validation. Bioanalysis 6(22), 2957-2963 (2014).

7 Hayes R, LeLacheur R, Dumont I et al. 9th GCC closed forum: CAPA in regulated bioanalysis; method robustness, biosimilars, preclinical method validation, endogenous biomarkers, whole blood stability, regulatory audit experiences and electronic laboratory notebooks. Bioanalysis 8(6), 487-495 (2016).

8 Cape S, Islam R, Nehls C et al. The $10^{\text {th }}$ GCC Closed Forum: rejected data, GCP in bioanalysis, extract stability, BAV, processed batch acceptance, matrix stability, critical reagents, ELN and data integrity and counteracting fraud. Bioanalysis 9(7), 505-516 (2017).

9 Lowes S, Jersey J, Shoup R et al. Recommendations on: internal standard criteria, stability, incurred sample reanalysis and recent 483s by the Global CRO Council for Bioanalysis. Bioanalysis 3(12), 1323-1332 (2011).

10 Boterman M, Doig M, Breda $M$ et al. Recommendations on the interpretation of the new EMA guideline on bioanalytical method validation by Global CRO Council for Bioanalysis (GCC). Bioanalysis 4(6), 651-660 (2012).

11 Sangster T, Maltas J, Struwe P et al. Recommendations on ISR in multi-analyte assays, QA/bioanalytical consultants and GCP by Global CRO Council for Bioanalysis (GCC). Bioanalysis 4(14), 1723-1730 (2012).

12 Lowes S, Boterman M, Doig M et al. Recommendations on bioanalytical method stability implications of co-administered and coformulated drugs by Global CRO Council for Bioanalysis (GCC). Bioanalysis 4(17), 2117-2126 (2012).

13 Hougton R, Gouty D, Allinson J et al. Recommendations on biomarker bioanalytical method validation by GCC. Bioanalysis 4(20), 2439-2446 (2012).

14 Lowes S, LeLacheur R, Shoup R et al. Recommendations on incurred sample stability (ISS) by GCC. Bioanalysis 6(18), 2385-2390 (2014).

15 European Medicines Agency. Committee for Medicinal Products for Human Use (CHMP). Guideline on Bioanalytical Method Validation. London, UK (2011). www.ema.europa.eu/docs/en_GB/document_library/Scientific_guideline/2011/08/WC500109686.pdf

16 US Department of Health and Human Services, US FDA, Center for Drug Evaluation and Research, Center for Veterinary Medicine. Guidance for Industry, Bioanalytical Method Validation. MD, USA (2001). www.fda.gov/downloads/Drugs/Guidance/ucm070107.pdf

17 Health Canada, Bureau of Pharmaceutical Sciences Therapeutic Products Directorate. Notice: Clarification of Bioanalytical Method Validation Procedures (2015). www.canada.ca/en/

18 Health Canada, Bureau of Pharmaceutical Sciences Therapeutic Products Directorate. Addendum to Notice: Clarification of Bioanalytical Method Validation Procedures (2016).

19 Song A, Lee A, Garofolo F et al. 2016 White Paper on recent issues in bioanalysis: focus on biomarker assay validation (BAV): (Part 2 - hybrid LBA/LCMS and input from regulatory agencies). Bioanalysis 8(23), 2457-2474 (2016).

20 Neubert H, Song A, Lee A et al. 2017 White Paper on recent issues in bioanalysis: rise of the hybrid LBA/LCMS immunogenicity 
assays: (Part 2 - hybrid LBA/LCMS biotherapeutics, biomarkers and immunogenicity assays and regulatory agencies' inputs). Bioanalysis 9(23), 1895-1912 (2017).

21 US Department of Health and Human Services, US FDA, Center for Drug Evaluation and Research (CDER) Center for Biologics Evaluation and Research (CBER) Center for Devices and Radiological Health (CDRH). Draft Guidance for Industry, Assay Development and Validation for Immunogenicity Testing of Therapeutic Protein Products Guidance for Industry (April 2016 Revision 1). www.fda.gov/downloads/Drugs/Guidances/UCM192750.pdf

22 European Medicines Agency. Committee for Medicinal Products for Human Use (CHMP). Guideline on Similar Biological Medicinal Products. London, UK (2014). CHMP/437/04 Rev 1. www.ema.europa.eu/docs/en_GB/document_library/Scientific_guideline/2009/09/WC500003517.pdf US Department of Health and Human Services, US FDA, Center for Drug Evaluation and Research, Center for Biologics Evaluation and Research. Scientific Considerations in Demonstrating Biosimilarity to a Reference Product (2015). www.fda.gov/downloads/drugsguidancecomplianceregulatoryinformation/guidances/ucm291128.pdf

24 European Medicines Agency. Committee for Medicinal Products for Human Use (CHMP). Guideline on Immunogenicity Assessment of Biotechnology-Derived Therapeutic Proteins. London, UK (2007). EMEA/CHMP/BMWP/14327/2006. www.ema.europa.eu/docs/en_GB/document_library/Scientific_guideline/2009/09/WC500003946.pdf of Monoclonal Antibodies Intended for in vivo Clinical Use. London, UK (2012). EMA/CHMP/BMWP/86289/2010. www.ema.europa.eu/docs/en_GB/document_library/Scientific_guideline/2012/06/WC500128688.pdf

US Department of Health and Human Services, US FDA, Center for Drug Evaluation and Research, Center for Veterinary Medicine. Draft Guidance for Industry, Bioanalytical Method Validation. MD, USA (2013). www.fda.gov/downloads/drugs/guidances/ucm368107.pdf Bioanalysis 4(18), 2213-2226 (2012).

28 Stevenson L, Rocci M, Garofolo F et al. 2013 White Paper on recent issues in bioanalysis: "hybrid" - the best of LBA \& LC/MS. Bioanalysis 5(23), 2903-2918 (2013).

Dufield D, Neubert H, Garofolo F et al. 2014 White Paper on recent issues in bioanalysis: a full immersion in bioanalysis (Part 2 hybrid LBA/LCMS, ELN \& regulatory agencies' input). Bioanalysis 6(23), 3237-3249 (2014).

30 Ackermann B, Neubert $\mathrm{H}$, Hughes $\mathrm{N}$ et al. 2015 White paper on recent issues in bioanalysis: focus on new technologies and biomarkers (Part 2 - hybrid LBA/LCMS and input from regulatory agencies). Bioanalysis 7(23), 3019-3034 (2015).

31 Jenkins R, Duggan JX, Aubrey A-F et al. Recommendations for validation of LC-MS/MS bioanalytical methods for protein biotherapeutics. AAPS J 17(1), 1-16 (2015).

32 Japanese Ministry of Health, Labour and Welfare. Guideline on Bioanalytical Method Validation in Pharmaceutical Development. Japan (2013).www.nihs.go.jp/drug/BMV/250913_BMV-GL_E.pdf

33 Welink J, Yang E, Hughes N et al. 2017 White Paper on recent issues in bioanalysis: rise of the hybrid LBA/LCMS immunogenicity assays: (Part 1 - LCMS: small molecules, peptides and small molecule biomarkers). Bioanalysis 9(23), 1895-1912 (2017).

CFDA. Announcement of the State Food and Drug Administration on carrying out self-examination and verification of drug clinical trial data (No. 117 of 2015). www.sfda.gov.cn/WS01/CL0087/124800.html

36 CFDA. Administration of General Administration of the People's Republic of China publicly solicits generic drug quality and efficacy of consistency assessment procedures and chemical generic drugs oral solid preparation consistency assessment reporting information requirements advice. www.sfda.gov.cn/WS01/CL0778/148660.html

CFDA. General Office of the General Public to seek generic quality and efficacy of consistency assessment of the site inspection and other guidelines www.sfda.gov.cn/WS01/CL1757/167816.html

Global CRO Council for Bioanalysis. www.global-cro-council.org 


\section{Conference Report Islam, Briscoe, Bower et al.}

Author affiliations

${ }^{1}$ Celerion, Lincoln, NE, USA

2PRA Health Sciences, Lenexa, KS, USA

${ }^{3}$ Covance, Chantilly, VA, USA

${ }^{4}$ Covance, Madison, WI, USA

${ }^{5}$ Covance, West Trenton, NJ, USA

${ }^{6} \mathrm{MPI}$ Research, Mattawan, MI, USA

${ }^{7}$ Atlanbio, Saint-Nazaire, France

${ }^{8}$ Pyxant Labs, Colorado Springs, CO, USA

9PPD Laboratories, Richmond, VA, USA

${ }^{10}$ Envigo, Somerset, NJ, USA

"PRA Health Sciences, Assen, The Netherlands

${ }^{12}$ Frontage Lab, Exton, PA, USA

${ }^{13}$ WuXi Apptec, Plainsboro, NJ, USA

1435 - Pharmacological Consultation, Bucharest, Romania

${ }^{15}$ ACDIMA Center for Bioequivalence \& Pharmaceutical Studies, Amman, Jordan

${ }^{16}$ AIT Bioscience, Indianapolis, IN, USA

${ }^{17}$ Alliance Pharma, Malvern, PA, USA

${ }^{18}$ Algorithme Pharma, Laval, Quebec, Canada

${ }^{19}$ Alturas Analytics, Moscow, ID, USA

${ }^{20}$ Anapharm Europe, Barcelona, Spain

${ }^{21}$ Axis Clinicals, Dilworth, MN, USA

${ }^{22}$ BASi, West Lafayette, IN, USA

${ }^{23}$ BioAgilytix, Durham, NC, USA

${ }^{24}$ BioPharma Services, Toronto, Ontario, Canada

${ }^{25}$ Biotrial Bioanalytical Services Inc., Laval, Quebec, Canada

${ }^{26}$ Charles River Laboratories, Reno, NV, USA

${ }^{27}$ Charles River Laboratories, Skokie, IL, USA

${ }^{28}$ CIRION Biopharma Research, Laval, Quebec, Canada

${ }^{29}$ CiToxLAB North America, Laval, Quebec, Canada

${ }^{30} \mathrm{CMIC}$, Hoffman Estates, IL, USA

${ }^{31}$ Covance, Salt Lake City, UT, USA

${ }^{32}$ CPR Pharma Services, Adelaide, Australia

${ }^{33}$ Eurofins Pharma Bioanalytics Services, Saint Charles, MO, USA

${ }^{34}$ Eurofins Pharma Bioanalytics Services, Moorpark, CA, USA

${ }^{35} \mathrm{GCC}$, Toronto, Ontario, Canada

${ }^{36}$ ICON Clinical, Whitesboro, NY, USA

${ }^{37}$ ICON Laboratory Services, Whitesboro, NY, USA

${ }^{38}$ Intertek, San Diego, CA, USA

${ }^{39}$ inVentiv Health, Quebec City, Quebec, Canada

${ }^{40}$ inVentiv Health, Princeton, NJ, USA

${ }^{41} \mathrm{KCAS}$ Bioanalytical \& Biomarker Services, Shawnee Mission, KS, USA

${ }^{42}$ Keystone Bioanalytical, North Wales, PA, USA

${ }^{43}$ LGC, Fordham, UK

${ }^{44}$ MicroConstants, San Diego, CA, USA

${ }^{45}$ Pharma Medica Research Inc., Mississauga, Ontario, Canada

${ }^{46}$ PPD Laboratories, Middleton, WI, USA

${ }^{47} Q^{2}$ Solutions, Ithaca, NY, USA

${ }^{48}$ Pharma Serv International, Bucharest, Romania

${ }^{49}$ Smithers Avanza, Gaithersburg, MA, USA

${ }^{50}$ SNBL USA, Everett, WA, USA

${ }^{51}$ Worldwide Clinical Trials, Austin, TX, USA

*Author for correspondence: wei@global-cro-council.org 